\title{
Metric Selection for GPU Kernel Classification
}

\author{
S.-KAZEM SHEKOFTEH, HAMID NOORI, MAHMOUD NAGHIBZADEH, and \\ HADI SADOGHI YAZDI, Department of Computer Engineering, Ferdowsi University of Mashhad, \\ Mashhad, Iran \\ HOLGER FRÖNING, Institute of Computer Engineering, Ruprecht-Karls University of Heidelberg, \\ Heidelberg, Baden-Wurttemberg, Germany
}

\begin{abstract}
Graphics Processing Units (GPUs) are vastly used for running massively parallel programs. GPU kernels exhibit different behavior at runtime and can usually be classified in a simple form as either "compute-bound" or "memory-bound." Recent GPUs are capable of concurrently running multiple kernels, which raises the question of how to most appropriately schedule kernels to achieve higher performance. In particular, coscheduling of compute-bound and memory-bound kernels seems promising. However, its benefits as well as drawbacks must be determined along with which kernels should be selected for a concurrent execution. Classifying kernels can be performed online by instrumentation based on performance counters. This work conducts a thorough analysis of the metrics collected from various benchmarks from Rodinia and CUDA SDK. The goal is to find the minimum number of effective metrics that enables online classification of kernels with a low overhead. This study employs a wrapper-based feature selection method based on the Fisher feature selection criterion. The results of experiments show that to classify kernels with a high accuracy, only three and five metrics are sufficient on a Kepler and a Pascal GPU, respectively. The proposed method is then utilized for a runtime scheduler. The results show an average speedup of $1.18 \times$ and $1.1 \times$ compared with a serial and a random scheduler, respectively.
\end{abstract}

CCS Concepts: $\bullet$ Theory of computation $\rightarrow$ Concurrency; $\bullet$ Computing methodologies $\rightarrow$ Supervised learning by classification;

Additional Key Words and Phrases: Classification, concurrency, feature selection, kernel metrics, resource utilization

ACM Reference format:

S.-Kazem Shekofteh, Hamid Noori, Mahmoud Naghibzadeh, Hadi Sadoghi Yazdi, and Holger Fröning. 2019. Metric Selection for GPU Kernel Classification. ACM Trans. Archit. Code Optim. 15, 4, Article 68 (January 2019), 27 pages.

https://doi.org/10.1145/3295690

\section{INTRODUCTION}

Traditionally, Graphics Processing Units (GPUs) were utilized for only performing graphic functions and displaying text and graphic information. However, nowadays GPUs are being used in servers, desktop, and even mobile devices. GPUs are expected to be employed for gaining more throughput

Authors' addresses: S.-Kazem Shekofteh; email: k.shekofteh@mail.um.ac.ir; H. Noori; email: hnoori@um.ac.ir; M. Naghibzadeh; email: naghibzadeh@um.ac.ir; H. S. Yazdi; email: h-sadoghi@um.ac.ir; H. Fröning; email: holger. froening@ziti.uni-heidelberg.de.

Permission to make digital or hard copies of all or part of this work for personal or classroom use is granted without fee provided that copies are not made or distributed for profit or commercial advantage and that copies bear this notice and the full citation on the first page. Copyrights for components of this work owned by others than the author(s) must be honored. Abstracting with credit is permitted. To copy otherwise, or republish, to post on servers or to redistribute to lists, requires prior specific permission and/or a fee. Request permissions from permissions@acm.org.

(C) 2019 Copyright held by the owner/author(s). Publication rights licensed to ACM.

1544-3566/2019/01-ART68

https://doi.org/10.1145/3295690

ACM Transactions on Architecture and Code Optimization, Vol. 15, No. 4, Article 68. Publication date: January 2019. 
for data-level parallel applications such as image processing, solving mathematician problems, molecular dynamics, simulations, and modeling [1].

A kernel is a concept in the context of parallel programming that uses GPUs, and in some forms, CPUs and FPGAs. It is a function specified by particular keywords and it is targeted to run on an accelerator instead of a general purpose processor during a parallel phase [2]. Threads are instances of a kernel in execution and similar to other codes, kernels can have different behaviors during runtime in terms of hardware resource utilizations.

Kepler-class and later NVIDIA GPUs are augmented with a "stream" capability that enables the execution of several kernels concurrently so that GPU resources may be utilized more efficiently $[3,4]$. This capability poses novel challenges of how to manage concurrent kernels and how to mix them so as to achieve the best performance. There is also the issue of whether to co-run applications on the CPU and GPU or not [5]. The current CUDA API [6] bestows the complete responsibility of controlling the concurrency of kernels to the programmer.

To prevent saturation of resources, knowledge of the resource usage of concurrent kernels is necessary; otherwise kernels may be launched concurrently but executed serially. An open problem is which kernels should be selected to run concurrently. One solution is to monitor the runtime behavior of kernels. CUPTI API [7] provides event and metric measurement over the entire execution of a GPU kernel and collects statistics about different resource usage for NVIDIA GPUs.

The results of the metric selection method can further be utilized by an online scheduler for better scheduling. The issue of resource usage is more severe in shared environments such as clouds [8]. Therefore, one of the main targets of the proposed method is the scheduling layer of such systems. There already exist different scheduling methods, such as [9-11]. However, they rely on modifications in hardware specifications [12] while our proposed method is applied at software level.

An online scheduler requires a certain amount of information about the runtime behavior of the kernels. In this regard, it has to read some metrics to classify the kernels. Since reading a metric requires a complete pass over a kernel execution, such as in CUPTI, forcing the scheduler to read more metrics further delays the scheduling decision.

The main contribution of this article is proposing a methodology to select the minimum number of metrics for a highly accurate classification of kernels at the least runtime overhead. Here, the term "runtime overhead" refers to the overhead of reading a metric during the runtime of a kernel. It is important that the classifier has as little runtime overhead as possible, as scheduling optimizations are only worthwhile if they contribute marginally to the overall execution time. To this end, the current study collects data from experiments on standard benchmarks, which are afterward processed by a machine learning method. Since it is a statistical method, this method is studied from both theoretical and experimental aspects. It does not need any binary code analysis and relies only on performance counters or metrics.

The contributions of this work are as follows:

- Proposing a novel method to select the minimum number of metrics for an accurate classification of kernels while minimizing the classification overhead.

-Evaluating the proposed method on two different architectures on real hardware showing different metric sets for each architecture. The results express that metric selection depends on the underlying architecture and it does not have a unified solution across all architectures.

-Using the results generated for each architecture to develop a scheduler to co-schedule different kernels and achieving performance improvement. The results emphasize that choosing a good set of metrics for kernel classification guides the scheduler to obtain performance improvement. 
The rest of the article is organized as follows. Section 2 provides a background on GPUs, programming model, and metric definition. Section 3 covers related work in this field. The proposed method for analyzing kernels and the feature selection method are presented in Section 4. The experiments and results are given in Section 5. Finally, Section 6 concludes the article and discusses future open works in this area.

\section{BACKGROUND AND MOTIVATION}

According to the usage of GPUs for current general purpose computing, an important research topic is resource management and the scheduling of kernels for different purposes [13-16]. Most of these works require observing the behavior of kernels either offline or online $[15,17]$.

Vendors provide limited information about the interior of GPUs although they have whitepapers to express general information about the architectures. There are also studies conducted on the reverse engineering of the complex GPU architecture and memory hierarchy $[18,19]$ via microbenchmarks. Some works, like Stargazer [20], propose an automated GPU performance exploration framework based on stepwise regression modeling. Similarly, Kerr et al. [21] evaluate a new set of metrics for GPU workloads and analyze the behavior of GPU programs.

In this section, a background on GPU architecture, programming models, feature selection methods, and kernel metric definitions is presented to facilitate understanding the context and concepts of the present article.

\subsection{GPU Architecture and Programming Models}

Although not fundamentally limited to this vendor, the current study focuses on NVIDIA GPUs. A GPU is generally composed of different components such as multiple Streaming Multiprocessors (SMs), memory controller units, and L2 caches. SMs and warps within it that are thread scheduling units have independent program counters. They are the part of the GPU that run CUDA kernels. Each SM includes resources such as processing cores, shared memory (a block-level memory accessible to all threads of the same block), and registers.

CUDA and OpenCL [22] are the most commonly used programming models. CUDA is supported on NVIDIA products while most GPUs from various vendors support OpenCL. In the following, CUDA is assumed as the programming model, but again it should be noted that the present work is programming language independent. Similar to CPU codes, various kernels running on a GPU have different resource requirements, thus leading to different categories, such as the ones mentioned in the Berkeley Dwarf taxonomy [23], quadrant-split model [24, 25], or the Roofline model [26]. In the simplest form, a kernel may belong to either a "compute-bound" or "memory-bound" class based on its ratio of memory transactions and the rate of issuing instructions [27].

Concurrent execution of kernels is a feature introduced in Kepler architecture and its successors by the means of stream capability. This allows GPU resources to be simultaneously shared among kernels. However, if this feature is not effectively employed, some resources might remain underutilized while a kernel is running [28]. Therefore, it is beneficial to allocate these unused resources to other kernels. Knowing which class the kernels belong to can help for more efficient co-scheduling of kernels using the stream capability.

\subsection{Kernel Metrics}

Via metrics, NVIDIA CUPTI library provides the programmer with information about the behavior of kernels at runtime. There are various numbers of metrics supported by different GPU architectures. Some familiar metrics are IPC (Instructions Per Cycle) and cache_hit_rate. There are several metrics in CUPTI that reflect resource utilization, e.g., dram_utilization, L2_utilization, 
Table 1. Hardware Configurations

\begin{tabular}{|l|c|c|}
\hline & System 1 & System 2 \\
\hline CPU & Core i7-4770K & AMD FX-9590 \\
\hline Main Memory & $8 \mathrm{~GB}$ & $8 \mathrm{~GB}$ \\
\hline GPU Model & GeForce GTX 760 & TITAN XP \\
\hline GPU Main Memory & $2 \mathrm{~GB}$ & $12 \mathrm{~GB}$ \\
\hline GPU CUDA Cores & 1152 & 3840 \\
\hline GPU SMs & 6 & 30 \\
\hline Operating System & Windows 7 & Ubuntu 16.4 \\
\hline
\end{tabular}

Table 2. List of Benchmarks for Collecting Metric Data: Numbers in the Paranthesses Represent Number of Different Input Sizes

\begin{tabular}{|c|c||c|c|}
\hline Benchmark Suite & Benchmark App. & Benchmark Suite & Benchmark App. \\
\hline & convolutionSeparable (4) & & Pathfinder (10) \\
& FDTD3d (16) & & bfs (16) \\
& quasirandomGenerator (11) & & backprop (8) \\
NVIDIA CUDA & scan (18) & & nn (12) \\
Sample SDK & matrixMul (20) & Rodinia & b+(4) \\
& mergeSort (14) & & srad (4) \\
& histogram (15) & & hotspot (12) \\
& sortingNetworks (18) & & \\
& vectorAdd (18) & & \\
\hline
\end{tabular}

$a l u \_f u \_u t i l i z a t i o n$, or metrics that correspond to the size of data transferred among the resources of a GPU, such as local_load_transactions, and dram_write_transactions.

\subsection{Basics of Classification and Feature Selection}

In the context of machine learning and statistics, there are two important concepts: classification and feature selection [29, Ch. 4]. Classification is an example of pattern recognition. Given a training set of data containing observations whose category membership is known, the problem is the identification of a new observation's category. The training set contains some samples with their features and feature values are the basis of designing the classifier. Hence, the number of features strongly influences the complexity of the classifier and the process of designing it. The process of extracting features necessary for accurate classification from the observed samples is called feature selection. In other words, feature selection can be considered as a mapping from an $\mathrm{N}$-dimensional space to a lower-dimensional feature space [29, Ch. 4].

\subsection{Motivation}

The presented study first visualizes the problem space and the need to reduce the number of features to design the classifier. In this regard, to collect different metric values, 239 experiments are conducted using benchmarks from the CUDA Sample SDK [30] and Rodinia Benchmark Suite [31]. The experiments are performed on a system whose configuration is shown in Table 1, column "System 1," which supports 94 CUPTI metrics.

The benchmarks for these experiments are listed in Table 2. For each benchmark application, all the kernels are run with different input sizes data and 94 metrics are collected. Some of the 


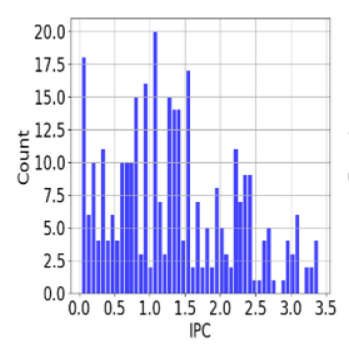

(a)

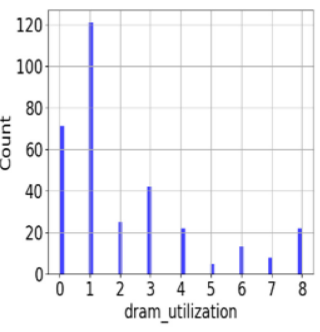

(b)

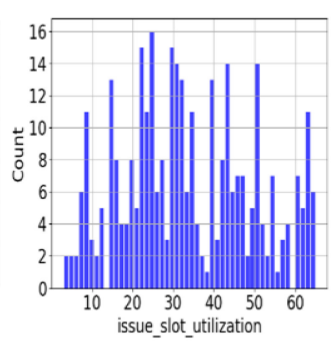

(c)

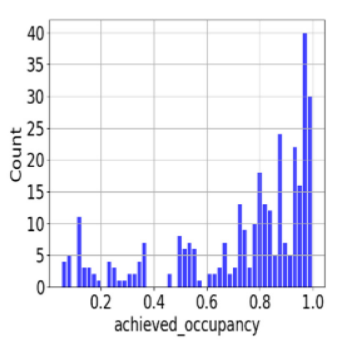

(d)

Fig. 1. Visualization of four collected metric data. Using a Kolmogorov-Smirnov test, the corresponding p-value is $0.873,0.639,0.792$, and 0.681 for (a), (b), (c), and (d), respectively, that accepts the hypothesis of being a normal or a half-normal distribution.

metrics are selected randomly in order to be visualized by a distribution histogram over training samples. The metrics are IPC, dram_utilization, issue_slot_utilization, and achieved_occupancy. Figure 1 presents the results. For each metric, a bar chart indicates the histogram of values in a corresponding range.

By using a Kolmogorov-Smirnov test [32] on data for each metric, the hypothesis of being a normal distribution at 5\%-10\% significance level was accepted. The $p$-value for each subplot (a), (b), (c), and (d) is $0.873,0.639,0.792$, and 0.681 , respectively. Therefore, they are considered as normal or half-normal distributions.

Figure 1 demonstrates that different metrics cover various ranges of values with different shapes of distributions. In Figure 1(a), the values of the IPC metric are in the range of [0,3.5] and also the average value for this feature is 1.3. On the other hand, the metric dram_utilization in Figure 1(b) has only integer values in the range of $[0,8]$ and a half-normal distribution shape. The metric issue_slot_utilization reports a distribution very close to a normal one in the range of $[3,65]$, while it can be observed in Figure 1(d) that achieved_occupancy shows an unbalanced distribution.

It is clear that some metrics may have similar distributions or a correlation with other metrics. Therefore, those correlated and similar metrics should be ignored and just one should be selected in order to simplify the design of the classifier. Besides, any metrics that cannot provide enough discriminative information should also be omitted. Feature selection methods make these decisions.

\section{RELATED WORK}

There exists research work focusing on solving the same or a similar problem in the area of kernel classification.

Karami et al. [33] propose a statistical performance prediction model for predicting the runtime behavior of kernels based on a regression model and a Principal Component Analysis (PCA) between events and runtime statistics. Built with training data, the Principal Component (PC) space analyzes the behavior of new kernels. Each new kernel is mapped into this space and, based on its location the developer makes a decision. Each area in the space indicates a category of kernels, e.g., memory-bound or compute-bound.

The proposed method by Pattnaik et al. [34] also employs some metrics for the purpose of kernel classification into either the GPU-PIM or GPU-PIC class, where PIM and PIC stand for processingin-memory and processing-in-core, respectively. However, the four static metrics used in [34] are those related to the underlying hardware and so are subject to change from hardware to hardware. Therefore, a given hardware may require reading three metrics in category I, while, for another hardware, five metrics must be read. Furthermore, the proposed method requires that the source 
Table 3. Comparison of Similar Kernel Classification Works

\begin{tabular}{|c|l|l|}
\hline & \multicolumn{1}{|c|}{ Pros } & \multicolumn{1}{c|}{ Cons } \\
\hline Lee [15] & - simple method of classification & $\begin{array}{l}\text { - manual classification } \\
\text { - no details about implementation }\end{array}$ \\
\hline Karami [33] & - applicable to online classification & - needs all metrics to construct PCs \\
\hline Jiao [35] & - fast decision for online classification & $\begin{array}{l}\text { - requires all configurations to be learned } \\
\text { - applicable to pairs of kernels only }\end{array}$ \\
\hline Pattnaik [34] & - Easy to adjust tuning parameters & $\begin{array}{l}\text { - manual metric selection dedicated to a } \\
\text { specific architecture }\end{array}$ \\
\hline
\end{tabular}

code be modified or compiled first by a source-to-source translation compiler to embed metrics in the kernel call.

Neural networks are employed by Jiao et al. [35] to build a power-performance model. The model selects an efficient combination of concurrent kernels in terms of performance per watt. Some manually selected metrics are collected from training benchmarks at different frequency levels of cores and memory so as to determine the most energy efficient working point.

In [15], Lee et al. categorize kernels into four types based on their patterns of performance behavior variations as the number of thread blocks assigned to each core increases. The results are used to schedule concurrent kernels. The categorization is completely manual and the proposed scheduler knows to which of the four categories an input kernel belongs.

$\mathrm{Wu}$ et al. [36] proposed a performance prediction model for estimating how a kernel behaves on a given GPU. This is achieved by utilizing previously obtained performance and power data for a collection of kernels and online gathered performance metrics for a new kernel. Unfortunately, this requires that all metrics be read online.

There is also some research, such as that by Muralidharan [37], in which supervised classification is utilized to select an optimal code variant. However, the training data is constructed based on a feature vector extracted from the source code. Some works, such as [38, 39], attempt to predict the program execution time for which the former uses neural networks and the latter employs piecewise polynomial regression as a black-box approach.

Majumdar et al. [40] also consider compute-bound and memory-bound classes while they propose other classes in terms of performance scaling. They evaluate the kernel behavior and how it scales when there are different GPU resources assigned to the kernel.

Li et al. [3] accomplish some energy and performance optimizations over concurrent kernels. They propose a symbiotic scheduling method to better reorder the kernel sequence for launching.

Since the idea of kernel classification is directly studied in some works, such as [15, 35], a comparison of the advantages and disadvantages of these works with those of the current work is provided in Table 3. However, the only study comparable with this work is [33] because it also covers a similar scope of kernel classification.

In order to design an online classifier for future runtime scheduling, it is necessary to select the metrics with the least overhead and the highest performance. It is also advantageous to have a self-tuning classifier to dynamically adapt to the behavioral changes of the kernel at runtime.

\section{PROPOSED KERNEL ANALYSIS METHOD}

This section discusses the proposed method for analyzing kernel metrics and also defines a novel method of classifying kernels into predefined classes. The classes are initially assumed to be disjoint as the terms "compute-bound" and "memory-bound" may suggest this discrimination. This is also in line with the literature. Therefore, the proposed method supports classification for disjoint sets.

ACM Transactions on Architecture and Code Optimization, Vol. 15, No. 4, Article 68. Publication date: January 2019. 


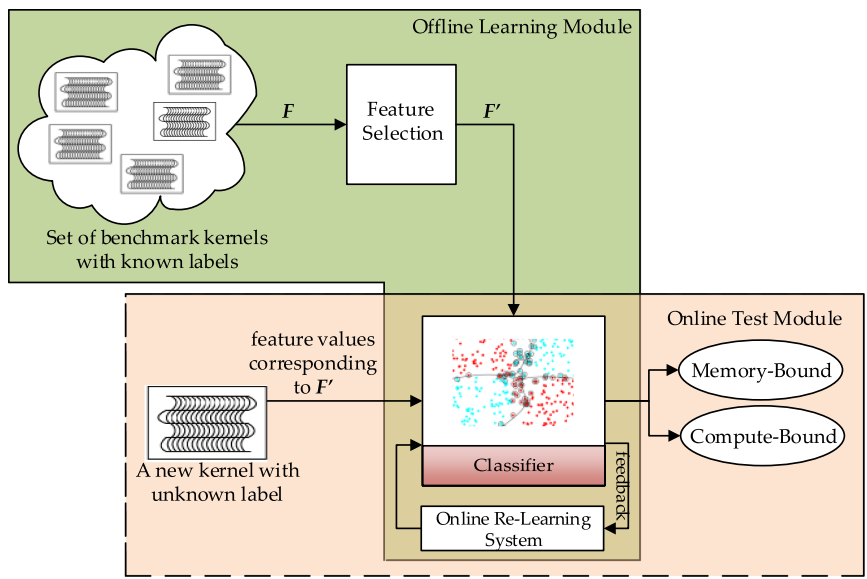

Fig. 2. The overall scheme of the proposed kernel classification system.

\subsection{Problem Statement}

The goal of this article is to identify a minimum number of the most effective metrics that by using them we would be able to classify the new input kernels as either memory-bound or computebound with a high accuracy.

A classifier is usually built based on the selected features of a sample dataset. The classifier is first learned via a training dataset and then used to answer the question to which class an input sample belongs. Therefore, a vital part of the process of designing the classifier is selecting the minimum set of appropriate features. This is crucial because, as the number of inputs to a classifier decreases, the design of the classifier and the classifier itself become simpler [29, Ch. 4].

In this context, kernel execution metrics are employed as input features. Hereinafter, the terms metric and feature may be interchangeably used. Consider vector $F=\left(f^{1}, f^{2}, \ldots, f^{d}\right)^{T}$ as the input feature vector, which has $d$ features. Subset $F^{\prime}=\left(f^{s_{1}}, f^{s_{2}}, \ldots, f^{s_{d^{\prime}}}\right)^{T}$ is the output of the feature selection method while attempting to minimize $d^{\prime}$, the number of selected features. $f^{i}$ is the $i$-th feature of the observed samples and $f^{s_{i}}$ represents the $i$-th selected feature from the initial feature vector $F$.

For new unlabeled kernels, only metric values corresponding to $F^{\prime}$ must be used as inputs to the classifier. There is also a feedback from the classifier when used at runtime to improve the learning system, especially the feature selection component. This process is shown in Figure 2.

\subsection{Feature Selection Approach}

Generally, feature selection methods are classified into three classes: filter-based, wrapper-based, and embedded methods [42]. For each class, there are several scoring functions that measure the quality or performance of a subset of input features. Figure 3 presents these three classes, including their scoring functions.

Filter methods are not capable of detecting possible interactions between features and they also suffer from a lack of an iterative evaluation of the selected subset. As their third drawback, filter methods do not take estimation accuracy into account. However, their advantage is having considerably enough speed for the learning phase, because scoring functions in this class, such as Fisher, are very fast. On the other hand, for conventional wrapper methods, no such fast scoring functions have yet been defined [41, 42]. 


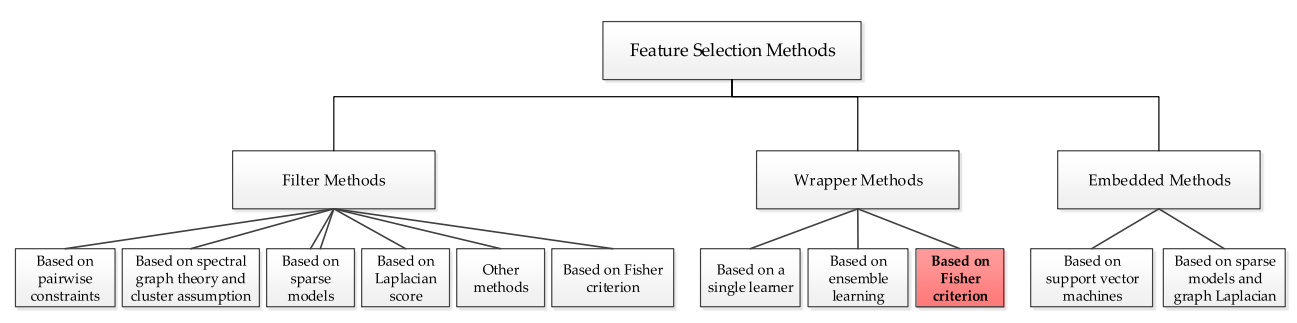

Fig. 3. The hierarchical structure of feature selection methods.

The problem is that the selected metrics for GPU kernels must be utilized during their runtime. Reading each selected metric has a runtime overhead. For this reason, metric minimization is critical for reducing the runtime overhead of the system. Although the wrapper method is an accuracybased feature selection method, it cannot meet this requirement since it is not fast enough. This is the inspiration behind utilizing the Fisher scoring function inside a wrapper method, as this combination inherits both the properties of high speed and accuracy-oriented behavior. This is achieved by the simplicity of updating an inverse covariance matrix with a recursive least square adaptive filter method.

Furthermore, embedded methods, such as SVM, have an optimization phase that results in a very time-consuming feature selection. On the other hand, methods like KNN [43], which are very fast at a very low cost, require a very large memory, which is also unsuitable for this topic and platform.

By employing a Fisher scoring function inside a wrapper feature selection method, the objectives can be reached in terms of

- detecting possible interactions among features;

- saving computation time and robustness to overfitting; and

-introducing the ability of simple and fast real-time re-learning.

Consequently, the proposal of a Fisher scoring function inside a wrapper-based feature selection method is novel. This expands the hierarchy shown in Figure 3 by adding a new leaf to the wrapperbased methods branch, as highlighted in this figure.

A wrapper-based method for feature selection is employed to find out the effective metrics for kernel classification [29, Ch. 4]. Assume that there are two classes of data, namely, $\omega_{1}$ and $\omega_{2}$. The goal is to project these two high-dimensional datasets onto vector $w$ to produce new datasets $y_{1}$ and $y_{2}$. Those datasets have a reduced number of features, such that a classifier based on new features is able to classify new data into two classes with an acceptable error rate. The block diagram of the feature extraction method is depicted in Figure 4 and the components are described in the following.

\subsubsection{Components of the Wrapper-Based Feature Selection.}

(1) Input $\boldsymbol{n} \times \boldsymbol{d}$-dimensional dataset: The input matrix $X$ is a set of $d$-dimensional samples $\boldsymbol{x}_{1} \ldots \boldsymbol{x}_{n}$, including $n_{i}$ of them in subset $D_{i}$, which are labeled as $\omega_{i}$. The matrix contains $d$ collected metrics from $n$ experiments. Each row represents a single experiment on a benchmark, while each column is the value of a metric. The input matrix $X$ is represented in Equation (1) where each $\boldsymbol{x}_{i}$ represents a feature vector in Equation (2).

$$
\begin{aligned}
X & =\left(x_{1}, x_{2}, \ldots, x_{n}\right)^{T} \\
x_{i} & =\left(x_{i}^{1}, x_{i}^{2}, \ldots, x_{i}^{d}\right)^{T}
\end{aligned}
$$




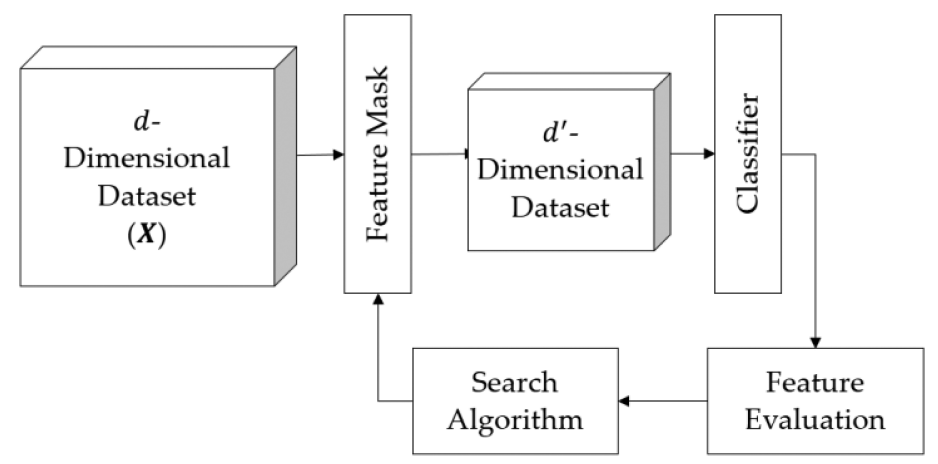

Fig. 4. The flowchart of the feature selection method in offline learning module.

(2) Feature Mask is a bitmap-style mask on the columns of the input feature vector. It can be considered as direction $\boldsymbol{w}$, on which input data is to be projected, and can be represented as

$$
\boldsymbol{w}=\left(\boldsymbol{w}^{1}, \boldsymbol{w}^{2}, \ldots, \boldsymbol{w}^{\boldsymbol{d}}\right)^{T},
$$

where each $w^{i}$ corresponds to a metric, e.g., IPC, alu_fu_utilization, or shared_efficiency and each $w^{i}$ can have a value of either zero or one.

(3) Projected $\boldsymbol{n} \times \boldsymbol{d}^{\prime}$-dimensional dataset is a matrix with a reduced number of metrics when compared to the input feature vector based on the feature mask, i.e., the column with a corresponding zero in the feature mask is removed from the new feature vector. $d^{\prime}$ is the number of ones in the feature mask $\left(d^{\prime}<d\right)$.

(4) Feature Evaluation: With a set of learning data in addition to a learning algorithm, minimum appropriate features are chosen to maximize the accuracy of the classification. The cost function in this search is a two-parameter function shown as Equation (4):

$$
\begin{aligned}
& f(\phi, r)=\frac{(\phi+\lambda r)}{1+\lambda} \\
& r=\left(1-\frac{d^{\prime}}{d}\right) \times 100,
\end{aligned}
$$

where $\phi$ (the performance of the classifier) is a function of the minimum Fisher criterion $\left[29\right.$, Ch. 4] and $r$ is the inverse ratio of the number of selected features $\left(d^{\prime}\right)$ to the total number of features $(d)$ as shown in Equation (5). $\lambda \in[0,1]$ is the controlling parameter which tunes the ratio of impact of two parameters $\phi$ and $r$ on the function value.

The goal is to find $(\phi, r)^{*}$ such that

$$
(\phi, r)^{*}=\arg \min _{\phi, r} f(\phi, r)
$$

(a) Calculation: In order to calculate $\phi$, the Fisher criterion must be measured. This criterion attempts to maximize the between-class scatter, i.e., the distance between the means of two classes, and to minimize the within-class scatter, which is equal to the sum of the variance of two classes defined in Equation (7).

$$
J=\frac{\left|\hat{\boldsymbol{m}}_{1}-\hat{\boldsymbol{m}}_{2}\right|}{\hat{\sigma}_{1}^{2}+\hat{\sigma}_{2}^{2}}
$$

where the numerator reflects the between-class scatter and the denominator represents the within-class scatter on the projection direction in the form of $y=w^{T} \boldsymbol{x}$ for 
the training set of $(x, y)$ in the form of $S=\left\{\left(\boldsymbol{x}_{i}, y_{i}\right), \forall i=1, \ldots, n\right\}$ where

$$
y_{i}=w^{1} x_{i}^{1}+w^{2} x_{i}^{2}+\ldots+w^{d} x_{i}^{d} .
$$

Hence, $\phi$ is calculated using Equation (10).

$$
\begin{aligned}
& J_{\min }=\min _{w} J(\boldsymbol{w}), \\
& \phi=e^{-\left(J_{\min } / \delta\right)},
\end{aligned}
$$

where $\delta$ is a scaling parameter. In order to find $J_{\min }$, it is beneficial to return to Equation (6) and take a close look. The mean of projected samples is shown as $\hat{\boldsymbol{m}}_{\boldsymbol{i}}$.

Hence, between-class scatter, which is the distance between the projected means, is calculated as

$$
\left|\hat{\boldsymbol{m}}_{1}-\hat{\boldsymbol{m}}_{2}\right|=\left|\boldsymbol{w}^{T}\left(\boldsymbol{m}_{1}-\boldsymbol{m}_{2}\right)\right| .
$$

The variance of the projected samples is defined as

$$
\hat{\sigma}_{i}^{2}=\sum_{y \in y_{i}}\left(y-\hat{\boldsymbol{m}}_{i}\right)^{2}=\boldsymbol{w}^{T} \sigma_{i} \boldsymbol{w} .
$$

Thus, the total within-class scatter is defined as

$$
\hat{\sigma}_{1}^{2}+\hat{\sigma}_{2}^{2}
$$

By defining the within-class scatter matrix as

$$
S_{w}=\sigma_{1}^{2}+\sigma_{2}^{2}
$$

it is proportional to the sample covariance for $d$-dimensional data. Previously defined in Equation (13), the total within-class scatter can finally be redefined as

$$
\hat{\sigma}_{1}^{2}+\hat{\sigma}_{2}^{2}=\boldsymbol{w}^{T} S_{w} \boldsymbol{w}
$$

Similarly, the total between-class scatter, i.e., the separation of the projected means, is defined as

$$
\left(\hat{\boldsymbol{m}}_{1}-\hat{\boldsymbol{m}}_{2}\right)^{2}=\boldsymbol{w}^{T}\left(\boldsymbol{m}_{1}-\boldsymbol{m}_{2}\right)^{2} \boldsymbol{w} .
$$

By defining the between-class scatter matrix as

$$
S_{B}=\left(\boldsymbol{m}_{1}-\boldsymbol{m}_{2}\right)\left(\boldsymbol{m}_{1}-\boldsymbol{m}_{2}\right)^{T},
$$

the total between-class scatter can at last be expressed as

$$
\left(\hat{\boldsymbol{m}}_{1}-\hat{\boldsymbol{m}}_{2}\right)^{2}=\boldsymbol{w}^{T} S_{B} \boldsymbol{w}
$$

In terms of $S_{w}$ and $S_{B}$, the Fisher criterion function $J($.$) over a sample direction w$ is defined as Equation (19):

$$
J(w)=\frac{w^{T} S_{B} w}{w^{T} S_{w} w} .
$$

The numerator and denominator reflect the between-class scatter and within-class scatter, respectively.

According to a generalized Rayleigh quotient [44], it is shown that vector $w$ maximizing $J($.$) must satisfy Equation (20):$

$$
S_{B} \boldsymbol{w}=\gamma S_{w} \boldsymbol{w}
$$

for some constant $\gamma$, which is a generalized eigenvalue problem. Briefly, the solution written for $\boldsymbol{w}$ optimizes $J($.$) as$

$$
\boldsymbol{w}=S_{w}^{-1}\left(\boldsymbol{m}_{1}-\boldsymbol{m}_{2}\right) .
$$


(b) Since the feature vector is a set of API metrics calculated using hardware counters, it is noteworthy that the less features chosen and used, the less overhead is incurred when collecting metrics during kernel runtime. Therefore, the number of features is used as a penalty for the evaluation function, which is considered as the second input parameter of the function. Since vector $v$ represents a subset of input feature vectors, the number of selected features equals the size of this subset, previously shown as $d^{\prime}$.

(5) Search Algorithm is the algorithm used for generating new feature masks. The overall approach is a sampling technique. The sampling can generally be done in two scenarios: a random search algorithm and an algorithm with prior knowledge. In the current work, since there is no prior knowledge about candidate results, a random search is used, i.e., in each iteration, a random subset of $n$ features is generated as the feature mask.

(5.1) Random Search Algorithm: Since the search space is usually extremely large ( $d$ dimensions) and exploring the whole space has an exponential time complexity of $O\left(\sum_{i=1}^{d}\left(\begin{array}{l}d \\ i\end{array}\right)=2^{d}\right)$, the algorithm employs a random-search-like approach to find the optimum point of this $d$-dimensional function. Because of the large search space, it is expected that there will be several sub-optimal and even optimal solutions at the end of each iteration of the algorithm. Obviously, if more time is spent for searching the space, skipping sub-optimal solutions and finding optimal solutions are more probable.

(5.2) Metaheuristic Search Algorithm: There are also other search methods like Genetic Algorithm, Hill Climbing, and Simulated Annealing. Although they are applicable to the proposed algorithm, but the focus of the proposed method is on the employed Fisher scoring function and not the search algorithm. However, three search algorithms including GA, HC, and SA are also evaluated and finally the best solutions are shown in results referred to in Section 5.

\subsection{Feature Selection Algorithm Details}

The algorithm consists of a main loop in which a random metric subset is selected and evaluated. The higher the accuracy in estimating the label, the higher the score. Moreover, the less subset metrics contained, the higher the score. The components of the feature selection method described in Section 4.2 are organized as a flowchart in Figure 5 and its implementation is also illustrated in Algorithm 1.

In line 1.1 of the algorithm, a random metric subset is generated. Then, the input dataset is projected on this subset, i.e., the metrics of the input dataset not existing in the subset are eliminated from the dataset. Lines 1.4-1.10 are the feature evaluation steps which measure classification accuracy (fitness score) for each random subset.

In order to study the time complexity of the algorithm and since the dimension of the matrices is a deterministic factor, the dimensions are shown in Table 4.

To find the time complexity of the whole algorithm, one must start with the time complexity of each section:

- Line 1.4: Calculating the covariance of two matrices, $\mathrm{XC}$ and $\mathrm{XM}$, is equal to calculating the covariance of the matrix generated by concatenating them, hereafter named X. Therefore, the time complexity for calculating the covariance of $\mathrm{X}$ is $O\left(n^{2} d\right)$.

- Lines 1.5 and 1.6: Since averaging the two matrices, $\mathrm{XC}$ and $\mathrm{XM}$, requires scanning through all the elements, an overall time of $O(n d)$ time is consumed. 


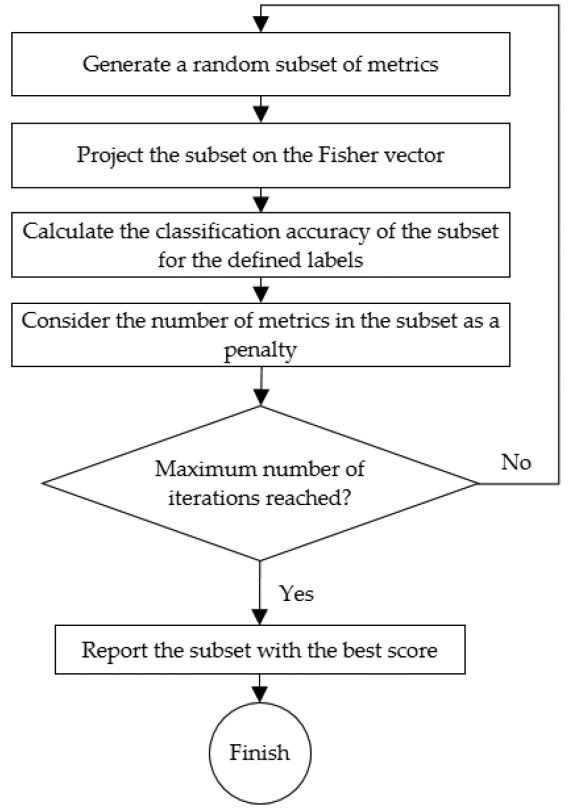

Fig. 5. The flowchart of Fisher-based feature selection algorithm.

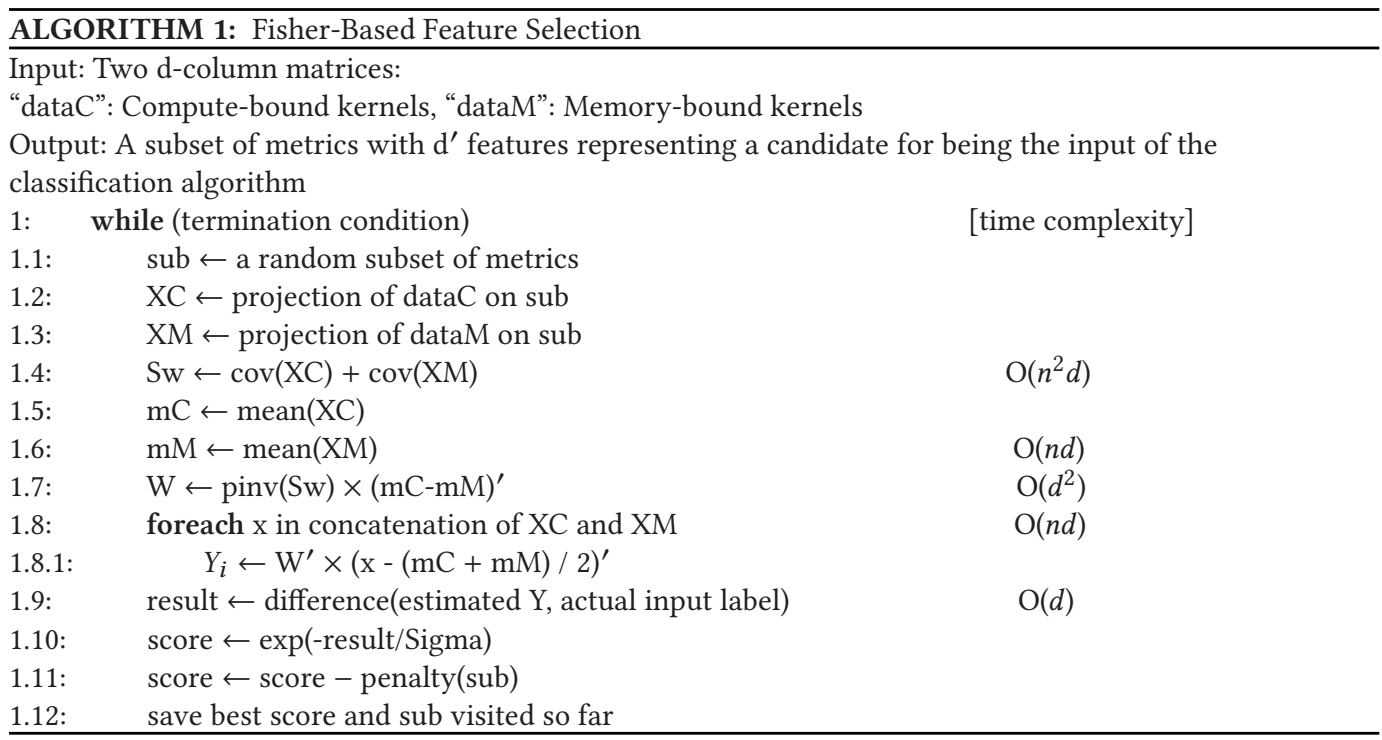

- Line 1.7: The time complexity for the multiplication of a $d \times d$ matrix with a $d \times 1$ matrix is $O\left(d^{2}\right)$.

-Line 1.8: The loop iterates $n$ times. In each iteration, the multiplication of a $1 \times d$ matrix with a $d \times 1$ matrix is calculated. Overall, this takes $O(n d)$ time.

-Line 1.9: The comparison of the estimated $Y$ matrix with actual input labels requires $O(d)$ time. 
Table 4. Diemnsions of the Matrices in Algorithm 1

\begin{tabular}{|c|c|}
\hline Matrix name & Dimension \\
\hline dataC & $n c \times d$ \\
\hline dataM & $n m \times d$ \\
\hline $\mathrm{Sw}$ & $d \times d$ \\
\hline $\mathrm{mC}, \mathrm{mM}$ & $1 \times d$ \\
\hline $\mathrm{W}$ & $d \times 1$ \\
\hline $\mathrm{Y}$ & $1 \times d$ \\
\hline
\end{tabular}

- Finally, the main loop should be studied. Since a termination condition is usually chosen as passing a predefined threshold for the score or a predefined fixed number of iterations, the worst case is scanning all the possible combinations of $d$ metrics. The number of iterations for the worst case is a member of $O\left(2^{d}\right)$. Otherwise, if a fixed number of iterations, namely, $R$, is set as the termination condition, then it is a member of $O(R)$.

In general, in the worst-case scenario of the main loop, the time complexity of the algorithm is $O\left(2^{n} n^{2} d+2^{n} d^{2}\right)$. Since $d$ is often considerably less than $n$, the time complexity for the worst case is $O\left(2^{n} n^{2} d\right)$. For a more realistic case, in which $R$ is set as an iteration count, the time complexity is $O\left(R n^{2} d\right)$. Although $R$ is a constant, since it may be set to a very large number comparable to $n$ and even larger than $n, R$ should be considered as a factor in calculating time complexity.

\subsection{Online Classifier Module}

After choosing the best metric subset, e.g., $S$ during the offline learning phase, the classifier is then constructed using $S$. When a kernel is to be classified at runtime, the same Fisher scoring function is used to project it onto the new space $S$ and finds out on which of the two classes compute-bound or memory-bound it will be mapped.

\section{EXPERIMENTS AND EVALUATIONS}

In this section, the details of the experimental setup and results are described.

\subsection{Preparation of Benchmarks}

Metric statistics are collected by profiling 460 experiments on 23 different kernels of 16 benchmark applications from NVIDIA CUDA Samples [30] and the Rodinia Benchmark Suite [31]. Different experiments are performed by varying the input data size of the benchmarks. All of the experiments are conducted on two different systems separately with the configurations specified in Table 1.

The first system has a Kepler-type GPU where the second one is equipped with a TITAN XP, with a Pascal architecture GPU. These two testbeds can show the results of the proposed method on two different architectures including an old generation and a new one.

Details of the experiments, including benchmark name and corresponding kernels, are described in Table 5. The column "Class" will be defined later in this section.

The "NVPROF-metrics all" command is applied to all of the above kernels and all the metrics are collected. Metrics, such as gld_transactions, gst_transactions, and flop_count_sp describe absolute values. This highly depends on the size of the input data. Therefore, it is suggested that all such metrics are eliminated from the dataset and only metrics with relative values are kept. Some examples of these remaining metrics are branch_efficiency, dram_read_throughput, sm_efficiency, dram_utilization, and IPC. By removing the mentioned metrics, their value and 
Table 5. Specification of the Workloads

\begin{tabular}{|c|c|c|}
\hline Benchmark App. & Kernels & Class \\
\hline \multirow{2}{*}{$\mathrm{b}+\left(\mathrm{R}^{*}\right)$} & findRangeK & $\mathrm{C}$ \\
\hline & findK & $\mathrm{C}$ \\
\hline \multirow{2}{*}{ convolutionSeparable $\left(\mathrm{N}^{*}\right)$} & convolutionColumnsKernel & $\mathrm{C}$ \\
\hline & convolutionRowsKernel & $\mathrm{C}$ \\
\hline FDTD3d (N) & FiniteDifferencesKernel & $\mathrm{C}$ \\
\hline nn (R) & Euclid & $\mathrm{M}$ \\
\hline \multirow{2}{*}{ quasirandomGenerator $(\mathrm{N})$} & inverseCNDKernel & $\mathrm{C}$ \\
\hline & quasirandomGeneratorKernel & $\mathrm{C}$ \\
\hline $\operatorname{scan}(\mathrm{N})$ & scanExclusiveShared2 & $\mathrm{M}$ \\
\hline \multirow{2}{*}{$\operatorname{srad}(\mathrm{R})$} & srad_cuda_1 & M \\
\hline & srad_cuda_2 & $\mathrm{M}$ \\
\hline hotspot (R) & calculate_temp & $\mathrm{C}$ \\
\hline kmeans (R) & kmeansPoint & M \\
\hline matrixMul $(\mathrm{N})$ & matrixMulCUDA & $\mathrm{C}$ \\
\hline \multirow{2}{*}{ mergeSort $(\mathrm{N})$} & generateSampleRanksKernel & $M$ \\
\hline & mergeSortSharedKernel & $\mathrm{C}$ \\
\hline sortingNetworks $(\mathrm{N})$ & bitonicMergeGlobal & M \\
\hline vectorAdd $(\mathrm{N})$ & vectorAdd & M \\
\hline hotspot3D & hotspotOpt1 & $\mathrm{C}$ \\
\hline \multirow{2}{*}{$\mathrm{BFS}$} & Kernel & \multirow{2}{*}{ M } \\
\hline & Kernel2 & \\
\hline \multirow{2}{*}{ Gaussian Elimination } & Fan1 & \multirow{2}{*}{$\mathrm{C}$} \\
\hline & Fan2 & \\
\hline histogram & histogram256Kernel & $\mathrm{M}$ \\
\hline transpose & transposeNaive & M \\
\hline
\end{tabular}

effect is still kept in other metrics. As an example, the value of gld_transactions is reflected in gld_transactions_per_request. Hereafter, the new metric set is called Rmetrics.

Since there is not enough reference information about kernels or their membership to either of the two classes (compute-bound or memory-bound), only 100 of the samples can be categorized into these classes, as specified in the "Class" column of Table 5. This is done according to the reports in related works [5, 10,21] and also execution analysis using NVIDIA Visual Profiler [7]. A "C" in this column stands for the compute-bound class and " $\mathrm{M}$ " represents the memory-bound class.

A cross-validation method of evaluation is utilized in which $70 \%$ of the samples are considered for training phase and the remaining 30\% are devoted to testing phase.

Definitions. There are some definitions employed in the following which are described here:

-Subset: a subset of the input Rmetric set.

-Score: a value in the range of [0-100] indicating the output of the feature evaluation function. The score represents how much the output can maximize the classification accuracy while minimize the number of metrics. An output with higher score is desired.

-Frequency: a percentage value in the range of [0-100] indicating the rate of how many times the specified subset is selected among all the generated subsets to the total number of generated subsets. 
Table 6. Metric Names and Abbreviations

\begin{tabular}{|c|c|c|c|c|c|c|c|}
\hline Abbrev. & Metric name & Abbrev. & Metric name & Abbrev. & Metric name & Abbrev. & Metric name \\
\hline SME & sm_efficiency & GLTP & $\begin{array}{c}\text { gld_transactions_ } \\
\text { per_request }\end{array}$ & ISU & issue_slot_utilization & GSE & gst_efficiency \\
\hline GLDRT & $\begin{array}{c}\text { gld_requested_ } \\
\text { throughput }\end{array}$ & GSTP & $\begin{array}{l}\text { gst_transactions_ } \\
\text { per_request }\end{array}$ & $\mathrm{ACO}$ & achieved_occupancy & ISI & issued_ipc \\
\hline GSTRT & $\begin{array}{c}\text { gst_requested_ } \\
\text { throughput }\end{array}$ & FLSE & flop_sp_efficiency & SYSMWT & $\begin{array}{c}\text { sysmem_- } \\
\text { write_throughput }\end{array}$ & $\mathrm{L} 2 \mathrm{U}$ & 12_utilization \\
\hline SMEI & $\begin{array}{c}\text { sm_efficiency_ } \\
\text { instance }\end{array}$ & FLDE & flop_dp_efficiency & DRU & dram_utilization & $\mathrm{CFU}$ & cf_fu_utilization \\
\hline SHRLTRQ & $\begin{array}{l}\text { shared_load_ } \\
\text { transactions_ } \\
\text { per_request }\end{array}$ & L2WT & 12_write_throughput & SHRLT & $\begin{array}{l}\text { shared_load__ } \\
\text { throughput }\end{array}$ & SHRST & $\begin{array}{l}\text { shared_store } \\
\text { throughput }\end{array}$ \\
\hline L2L1T & $\begin{array}{l}\text { 12_l1_read_ } \\
\text { throughput }\end{array}$ & L2RT & 12_read_throughput & INSRO & inst_replay_overhead & & \\
\hline
\end{tabular}

\subsection{Feature Selection Results}

Since the runtime of the algorithm's evaluation section (lines 1.2-1.10) for each candidate subset is long, the termination condition is set to 5,000 iterations. The experiments are repeated 100 times with the results varying each time, i.e., each output consists of metrics different from the previous ones. The subsets include four to seven metrics. This may be due to a very large search space. Although there are several subsets with $100 \%$ estimation accuracy, the penalty incurred by the number of metrics leads to lower scores.

In order to reduce the penalty, more time is devoted to running the algorithm with many more iterations to improve the exploration of the search space. The termination condition of the main loop is increased to 500,000 iterations and the experiments are repeated 100 times. In these experiments, there are only a limited number of solutions representing that almost all the search space is explored.

In the following, the metric names are abbreviated for short representation, as defined in Table 6 .

Since the results on the two testbeds in Table 1 are different, they are evaluated in different subsections as follows.

5.2.1 Results on Kepler. There are 107 metrics supported by NVIDIA GTX760. Using the strategy described in Section 5.1, by eliminating 60 absolute-valued metrics, 47 metrics remain as the input features of the proposed method.

The output subsets show $99.9074 \%$ estimation accuracy with only three metrics. This accuracy is high enough to consider three metrics as the desired minimum number of features. However, with four metrics, accuracy reaches $100 \%$.

The results are illustrated in Table 7, in which the score of all selected subsets is 90.4197 with no other subset with a higher score. The frequency of each subset is shown in the second column. The frequency is the ratio of observing a solution in 100 iterations, i.e., the first subset generated $21 \%$ on average. Having several possible solutions is a positive result, as some platforms may not support all of the metrics.

In order to further reduce the number of metrics even more and observe the impact of each single one, some complementary experiments are conducted for each metric in each row of Table 7. In other words, the main loop is run by substituting the sub variable (in Algorithm 1) with the selected metric instead of generating it randomly.

For example, for the first row, which is \{GLDRT, L2L1T, DRU\}, three experiments are performed on each of the GLDRT, L2L1T, and DRU metrics. The aim of this experiment is to evaluate the 
Table 7. Results of the Feature Selection Algorithm on GTX 760 in Terms of Frequency of Repetition of the Resulted Subsets over Different Iterations

\begin{tabular}{|c|c|}
\hline selected subset metrics & frequency \\
\hline \{GLDRT, L2L1T, DRU & $21 \%$ \\
\hline \{L2L1T, SHRST, DRU $\}$ & $17 \%$ \\
\hline$\{$ SME, INSRO, L2U & $17 \%$ \\
\hline$\{$ SHRLT, SYSMWT, DRU $\}$ & $15 \%$ \\
\hline$\{$ L2L1T, SHRLTRQ, DRU & $13 \%$ \\
\hline \{SME, INSRO, L2U & $10 \%$ \\
\hline \{GLDRT, SHRLTRQ, DRU $\}$ & $7 \%$ \\
\hline
\end{tabular}

All results have the same score 90.41 .

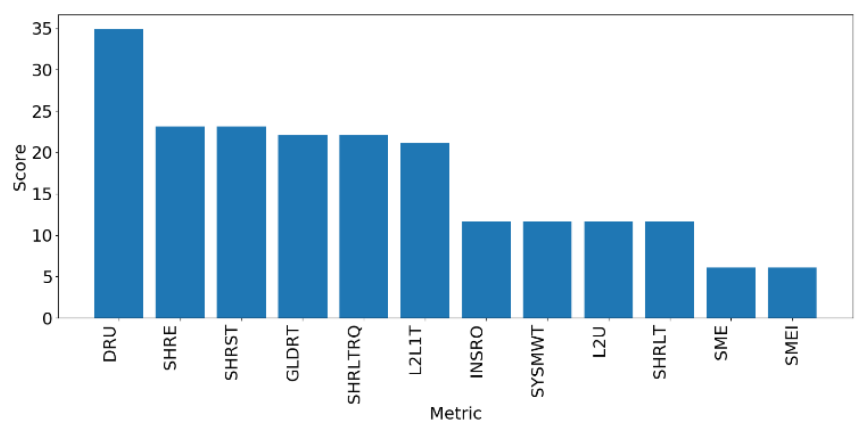

Fig. 6. Classification score on GTX 760 when using only one metric from the set of selected metrics in Table 7 .

efficiency of using just one metric instead of all the metrics in a subset. None of these metrics can individually provide sufficient accuracy and hence cannot be a satisfactory candidate. The score of each single metric in the best case, which is dram_utilization is not higher than 34.89 . The results are shown in Figure 6.

We also study two-metric subsets of metrics for evaluation. According to Table 7, since there are 55 different two-subset combinations of metrics, evaluations are performed on the combinations of the metrics for each row of Table 7. For example, for the first selected subset, which is \{GLDRT, L2L1T, DRU\}, three pairs are evaluated as \{GLDRT, L2L1T\}, \{GLDRT, DRU\}, and \{L2L1T, DRU\}. The results are given in Figure 7, where, for each row in Table 7, three bars of the same color are drawn. Each bar shows the score of a two-metric pair selected from that row. The maximum score for these experiments is 82.71 , which is a lower score compared to that of the three-metric pairs.

In some cases, however, the score of a two-metric subset may be less than the score of each of its single metrics, such as with [GLDRT, L2L1T]. This is due to the fact that when the number of metrics doubles, the value of $r$ decreases by a factor of 2 as well. Therefore, a greater penalty is considered for the score.

Since the best results are delivered by the three-metric subsets in Table 7, it is clear that increasing the number of candidate metrics will result in only an increase in the runtime overhead and, hence, a decrease in the score. To recall Equation (4), since $\phi$ is 100 for all the three-metric subsets in Table 7, adding a metric to a subset will not increase $\phi$ even though it decreases $r$, which results in a lower overall score. 

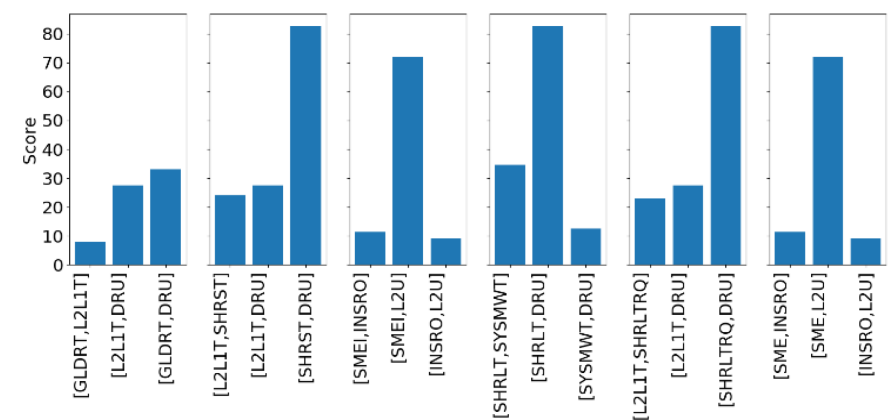

Fig. 7. Two-metric classification score: each sub-chart corresponds to one row of Table 7 on GTX 760.

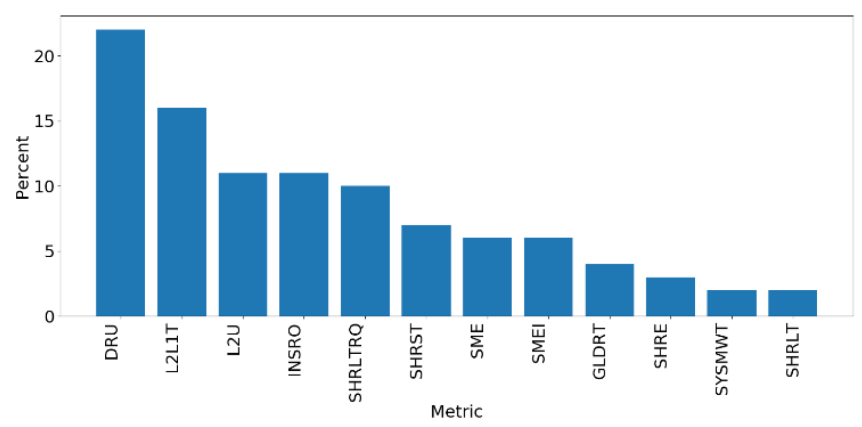

Fig. 8. Relative distribution of metrics in selected feature subsets on GTX 760 representing the relative importance of each metric among other metrics in the set of selected metrics in Table 7.

It is evident from Table 7 that high-frequency metrics are memory related, such as dram_utilization, $12 \_l 1 \_r e a d \_t h r o u g h p u t$, and $12 \_u t i l i z a t i o n$. On the other hand, there are other metrics such as inst_replay_overhead and sm_efficiency that are related to compute units.

In the next phase, these three-metric subsets are merged into a single set resulting in a 10-feature dataset instead of the initial 47-feature one. In other words, the input dataset is projected on these 10 features and the algorithm is run on the new 10-feature dataset. The results are the same for the new dataset. Besides, in this phase, the frequency of the metrics in the subsets is measured, as seen in Figure 8. In other words, dram_utilization is the most frequent metric.

5.2.2 Results on Pascal. To show the applicability of the proposed method to different hardware and architectures, the same experiments are conducted on a NVIDIA TITAN XP GPU (Pascal class) that supports 162 CUPTI metrics. Similar to previous experiments, 69 absolute-valued metrics are eliminated and the remaining 93 metrics are used to train the proposed classifier.

In order to achieve a classification estimation accuracy of more than $90 \%$, the minimum required number of metrics is five. The corresponding subset is \{gst_requested_throughput, sm_efficiency flop_sp_efficiency, flop_dp_efficiency\}. However, there are several subsets with six and seven metrics with average estimation accuracy of $92 \%$ and $97 \%$, respectively. In this device, two metrics flop_dp_efficiency and flop_sp_efficiency are the most frequent ones in the selected subsets. The results are shown in Table 8.

\subsection{Discussion on the Results}

Comparing Figure 8 with Figure 9, one can interpret that for a device like GTX 760 with more memory considerations, the most important metrics used for kernel classification are those related to 
Table 8. Results of the Feature Selection Algorithm on TITAN XP

\begin{tabular}{|c|c|}
\hline Selected subset metrics & EA $^{*}$ \\
\hline \{GSTRT,SME,ACO,FLDE,FLSE & $90.2 \%$ \\
\hline \{GSTP,L2RT,GSE,ISU,FLDE,FLSE & $92.5 \%$ \\
\hline \{GLTP,GSTP,GSE,ISU,CFU,FLDE,FLSE & $97.1 \%$ \\
\hline \{GLTP,GSTRT,SHRST,ISU,FLDE,FLSE,DRU\} & $97.3 \%$ \\
\hline \{GLTP,GSTP,GLDRT,SHRST,GSE,FLDE,FLSE & $96.4 \%$ \\
\hline \{GLTP,L2WT,GSE,ACO,ISU,CFU,FLDE,FLSE\} & $96.9 \%$ \\
\hline
\end{tabular}

${ }^{*}$ EA: Estimation Accuracy.

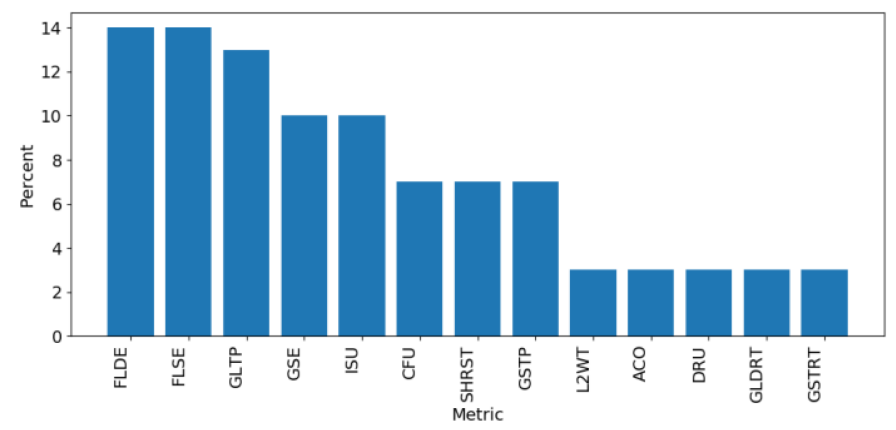

Fig. 9. Relative distribution of metrics in selected feature subsets on TITAN XP.

memory subsystems such as dram_utilization, while metrics corresponding to compute resources such as flop_dp_efficiency and flop_sp_efficiency are more determinant factors for TITAN XP.

On the other hand, in GTX 760, the memory-related metrics as shown in Table 7 can be sufficient for kernel classification, while in TITAN XP, a combination of both memory-related and computerelated metrics are required to this end as it is observable in Table 8.

To sum up, despite the number of metrics supported by hardware and the CUPTI library, which was previously shown as $d$ in Section 4.2, the proposed metric selection method can be used to design an online classifier with an order of magnitude of $O\left(f\left(d^{\prime}\right)\right)$ time for the classification process where $d^{\prime} \ll d$. This is due to two factors: (1) the process of reading $d^{\prime}$ metrics, which is a member of $O\left(f_{1}\left(d^{\prime}\right)\right)$, and (2) the process of mapping $d^{\prime}$ values to the new space, which is also a member of $O\left(f_{2}\left(d^{\prime}\right)\right)$.

\subsection{Instrumentation Penalty}

For better understanding of the overhead penalty, a function of the parameter $r$ in Equation (5), Figure 10 presents the average overhead penalty for reading different numbers of metrics at runtime. According to Equation (5), $r$ is the inverse ratio of the number of selected features $\left(d^{\prime}\right)$ to the total number of features $(d)$. Therefore, the overhead penalty is a function of $r$ that is shown as $P(r)$. The table provides the results only for one to seven metrics since if all available metrics are shown, the table will have too many rows.

In Figure 10, one can see that the overhead penalty increases at a constant rate as the number of read metrics increases.

It is assumed that there are $n$ metrics requiring $t_{1}$ units of time to be read and $m$ metrics requiring $t_{2}$ units of time to be read. Since $t_{2}$ is a multiple of $t_{1}$, it can be considered as $t_{2}=\alpha t_{1}$ (as the overhead time is directly affected by $\alpha$, i.e., number of kernel re-runs). The average overhead time for reading 


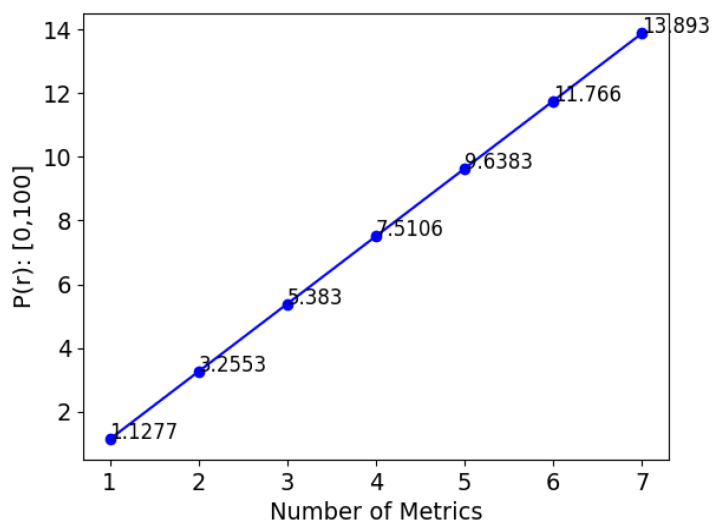

Fig. 10. The proportionality of relative overhead penalty $(P(r))$ to the number of metrics.

one metric is represented by $t_{1 m}$ and is calculated as

$$
t_{1 m}=\frac{n t_{1}+m t_{2}}{n+m}=\frac{n t_{1}+\alpha m t_{1}}{n+m}=\frac{n+\alpha m}{n+m} t_{1} .
$$

The average overhead time for reading two metrics, $t_{2 m}$, is calculated as

$$
t_{2 m}=\frac{n m\left(t_{1}+t_{2}\right)+\left(\begin{array}{c}
n \\
2
\end{array}\right) 2 t_{1}+\left(\begin{array}{c}
m \\
2
\end{array}\right) 2 t_{2}}{n m+\left(\begin{array}{c}
n \\
2
\end{array}\right)+\left(\begin{array}{c}
m \\
2
\end{array}\right)}=\frac{n m+n \alpha m+2\left(\begin{array}{c}
n \\
2
\end{array}\right)+2 \alpha\left(\begin{array}{c}
m \\
2
\end{array}\right)}{n m+\left(\begin{array}{c}
n \\
2
\end{array}\right)+\left(\begin{array}{c}
m \\
2
\end{array}\right)} t_{1},
$$

where three terms in the numerator represent three different cases of two metrics: (1) one from $n$ metrics and one from $m$ metrics requiring $t_{1}+t_{2}$ units of time, (2) both from $n$ metrics requiring $2 t_{1}$ units of time, and (3) both from $m$ metrics requiring $2 t_{2}$ units of time.

Therefore, to compare $t_{2 m}$ with $t_{1 m}$, their corresponding coefficients of $t_{1}$ must be compared. $A$ and $B$ are defined as follows, such that $t_{1 m}=A t_{1}$ and $t_{2 m}=B t_{1}$ :

$$
\begin{aligned}
& A=\frac{n+\alpha m}{n+m}, \\
& B=\frac{n m+n \alpha m+2\left(\begin{array}{c}
n \\
2
\end{array}\right)+2 \alpha\left(\begin{array}{c}
m \\
2
\end{array}\right)}{n m+\left(\begin{array}{c}
n \\
2
\end{array}\right)+\left(\begin{array}{c}
m \\
2
\end{array}\right)}=2 \frac{n m+n \alpha m+2\left(\begin{array}{c}
n \\
2
\end{array}\right)+2 \alpha\left(\begin{array}{c}
m \\
2
\end{array}\right)}{2 n m+n^{2}-n+m^{2}-m} .
\end{aligned}
$$

Then

$$
\frac{t_{2 m}}{t_{1 m}}=\frac{B}{A}
$$

To prove that $B=2 A$, it is necessary to show that

$$
B=2 \frac{n+\alpha m}{n+m} .
$$

By merging Equation (24), Equation (25), and Equation (27), it is demonstrated that

$$
\frac{n m+n \alpha m+2\left(\begin{array}{l}
n \\
2
\end{array}\right)+2 \alpha\left(\begin{array}{c}
m \\
2
\end{array}\right)}{2 n m+n^{2}-n+m^{2}-m}=\frac{n+\alpha m}{n+m} .
$$

Since the cross-multiplying of Equation (28) is correct, it is proven that $B=2 A$.

The analysis can be similarly extended for more than two types of metrics that results in

$$
t_{k m}=\mathrm{k} t_{1 m} \text {. }
$$




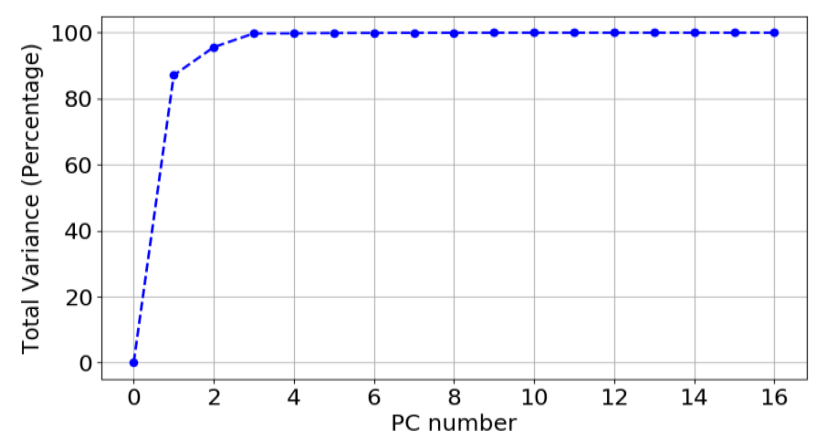

Fig. 11. Total variance explained by different PCs.

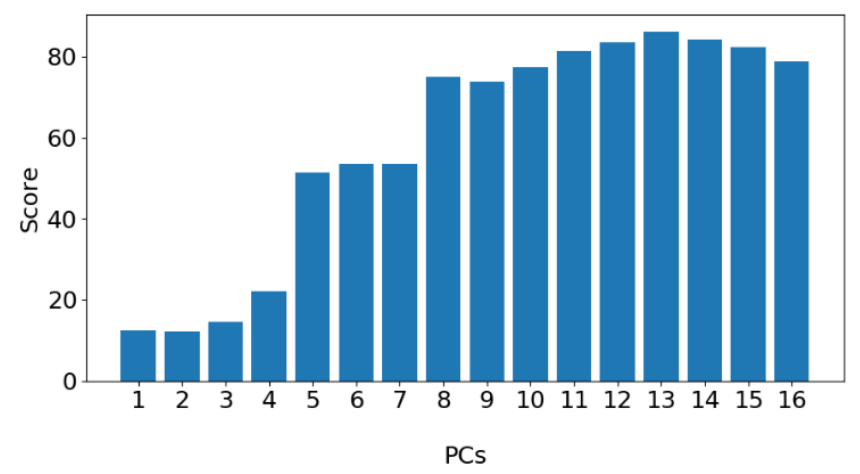

Fig. 12. The score of the first 16 PCs when being run with the proposed Fisher criteria.

\subsection{Comparing with PCA}

As mentioned in Section 3, Karami et al. [33] propose a method of kernel classification based on PCA and regression methods. It should be noted that for PCA, all metrics are required to participate in the extraction of principle components. Therefore, the overhead will be very high for online classification. However, our proposed method takes this into account since the number of required metrics is minimized during the learning phase.

As previously shown in Equation (4), there is a two-parameter function $f$ in the proposed method. This function tries to maximize both parameters to obtain a better score. The second parameter, shown as $r$, represents the goodness corresponding to the overhead of reading metric values. According to Equation (5), for PCA, $r$ is likely to be zero since $d^{\prime}$ equals $d$ in both the learning and testing phases, if all the metrics are included in the calculation of principle components.

Although the method proposed in [33] provides enough classification accuracy, it does not seem to be beneficial for online classification due to high overhead. The currently proposed method, however, provides both high accuracy and lower overhead. On the other hand, PCA shows satisfactory results for this purpose. Figure 11 illustrates the percentage of the total variance explained by each PC. It is obvious that the first three components will provide enough estimation accuracy.

In an interesting experiment, PCA is applied and then the output is used by the Fisher criteria. The final output contains the new observation coordination in the new PC space. It is clear that the first new feature, which is the first PC, holds the most meaningful information even though it reports only 13.6\% estimation accuracy and a score of 12.319. As defined in Equation (4), Figure 12 presents the corresponding score for the first PC, the first two PCs, and so on until the first 13 PCs 
when the score reaches the maximum of 86.137. Subsequent PCs can provide the same estimation accuracy but with a lower score due to the greater number of PCs used as in Equation (5).

To sum up, since PCA requires all metrics to be included in the classification process, the PCAbased online classifier runtime will take an order of magnitude of $O(f(d))$, which is a function of the number of metrics supported by hardware and the CUPTI library. All the while, our proposed method can offer an $O\left(f\left(d^{\prime}\right)\right)$ classification process time. This time complexity for PCA-based classification is due to two factors: (1) the process of reading $d$ metrics, which can be considered as a member of $O\left(f_{1}(d)\right)$ and (2) the process of mapping $d$ values in the new space, which is also a member of $O\left(f_{2}(d)\right)$.

\subsection{Concurrent Kernel Scheduling}

The current study conducts a critical experiment demonstrating the application of the proposed metric selection method for scheduling of concurrent kernel execution. To this end, the available "stream" capability of modern GPUs, which enables concurrent execution of multiple kernels via hardware queues, is used [3]. Briefly, all kernel launches and commands submitted to one stream can be serviced concurrently with other streams.

According to our observations, the stream capability is more effective in terms of performance when the two concurrent (co-scheduled) kernels are from different types (i.e., one from computebound and the other from memory-bound). Our observations show that when two kernels are of the same type (either compute-bound or memory-bound), the stream capability is not effective as the kernels are from different types.

A runtime scheduler is developed within which the proposed method of metric selection and the corresponding classification method are used as the kernel classifier component. The classifier is responsible for classifying an input kernel into either the "memory-bound" or the "computebound" class. To enhance the performance, the scheduler tries to overlap the execution of a memory-bound kernel with a compute-bound one. The goal is similar to work proposed by $\mathrm{Li}$ et al. [3] to fully utilize the GPU. At each scheduling phase, a compute-bound kernel, namely, $K_{c}$, and a memory-bound kernel, namely, $K_{M}$, are chosen from a set of input kernels and are launched in different streams. Hereafter, this scheduler is called the Class-Based Scheduler (CBS).

To evaluate the efficiency of this proposed scheduler, a RS is also designed which randomly chooses different kernels without considering which classes they belong to for overlapping their execution. In other words, the scheduler randomly chooses two kernels and launches them in different streams. This scheduler is hereafter called the Random Scheduler (RS).

In order to study the effect of selecting correct and sufficient metrics, we also consider a CBS using only dram_utilization (DRU) metric. The scheduler uses this metric to classify kernels.

Finally, for a given set of kernels, we evaluate all possible combinations to find the optimal scheduling for a set of kernels. This is another case for comparing the proposed method with. As a paragon, the results of optimal scheduling and serial scheduling can be used to define the upper bound and lower bound of the proposed method.

To demonstrate the efficiency of the proposed metric selection method, the experiments including CBS, RS, DRU, and optimal scheduling are compared over a different set of benchmarks. Five benchmarks are selected to be included in these experiments: vectorAdd (vd), matrixMultiplication (mm), matrixTranspose (tr), hotspot (hs), and histogram (htg). In order to better evaluate the performance improvement, only experiments with at least four kernels are considered since with less kernels, there is not enough opportunity for the scheduler to choose among kernels.

Since there is no commonly agreed set of concurrently running kernels, to avoid using randomized kernel streams, these kernels and combinations are chosen including very suitable candidates as well as less suitable candidates. 


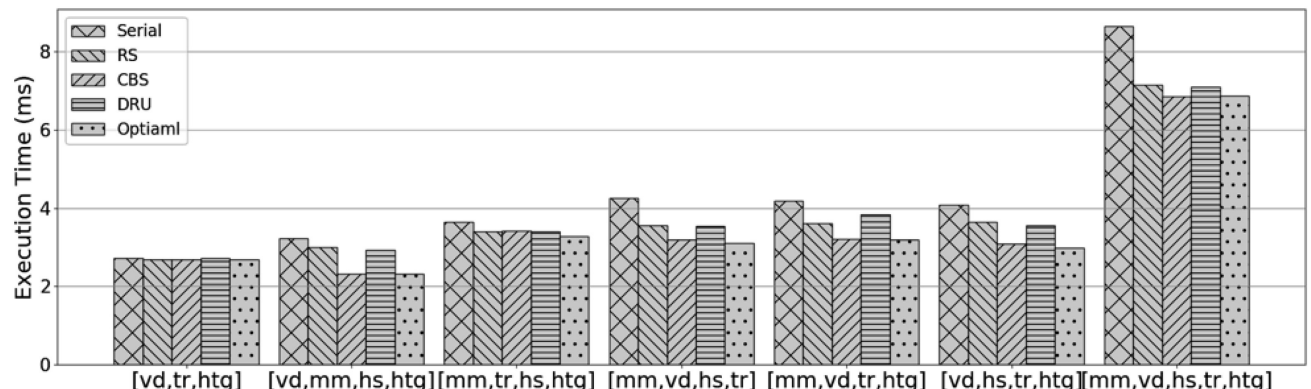

Kernel Set

(a)

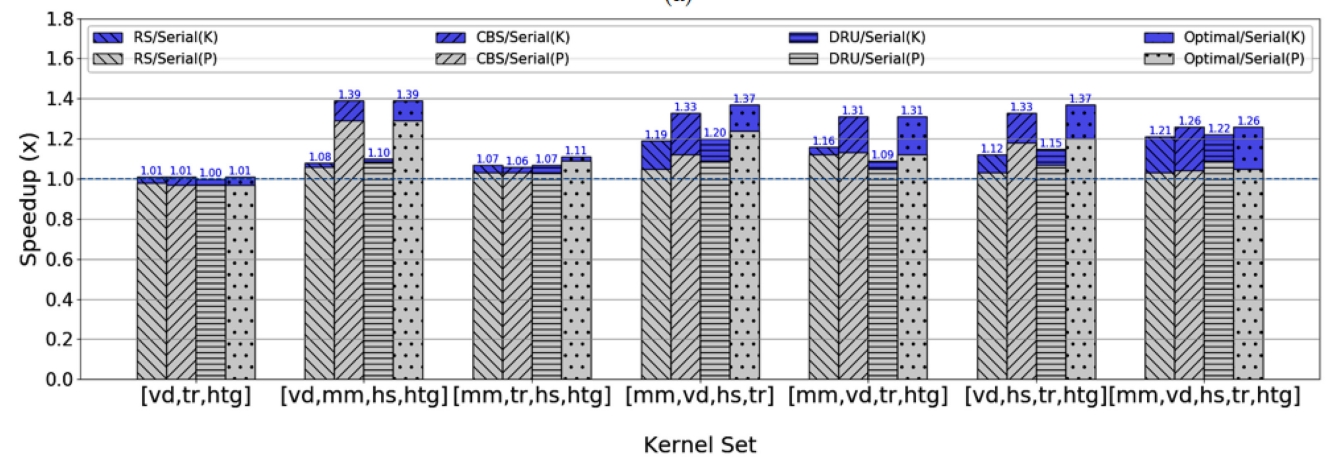

(b)

Fig. 13. The results of utilizing the proposed method of kernel classification in a scheduler (CBS) compared with a random scheduler (RS), optimal scheduler, dram_utilization-based scheduler, and serial execution. (a) Comparing execution time in each scheduling method. (b) Comparing achieved speedup over serial execution in each scheduling method. The bars marked with $(K)$ depict the speedup of the kernel execution over kernel serial execution while $(P)$ represents the speedup of the whole program execution over serial execution.

In addition, serial execution for each experiment is also considered as the basis for comparisons. In all cases including serial, CBS, RS, DRU, and optimal, the benchmarks are chosen such that the number of thread blocks is large enough to provide sufficient threads for the GPU. Each experiment is executed 100 times and the average is considered as the result. The results are given in Figure 13.

Figure 13 contains two sub-figures (a) and (b). Figure 13(a) represents the execution time of each experiment while Figure 13(b) depicts the speedup for four experiment cases. Each bar in Figure 13(b) consists of two parts: the blue bar (that includes the gray also) shows the speedup corresponding to the kernel execution time while the gray part represents the speedup of the whole execution time including kernel time, memory copies, and scheduling overhead.

As seen in Figure 13, CBS provides more speedup on average, compared to a RS relative to serial execution. The speedup, however, varies in different experiments, e.g., for the kernel set [mm,tr,hs,htg], only $6 \%$ speedup is achieved by CBS, while for the kernel set [vd,mm,hs,htg], speedup is near to $40 \%$. The speedup for the last experiment including all five kernels is also considerable, which shows promising results for utilizing the proposed method of kernel classification inside a scheduler.

The first experiment set includes three memory-bound kernels. This case is also important since it does not contain any compute-bound kernel. In this case, there is no significant performance 

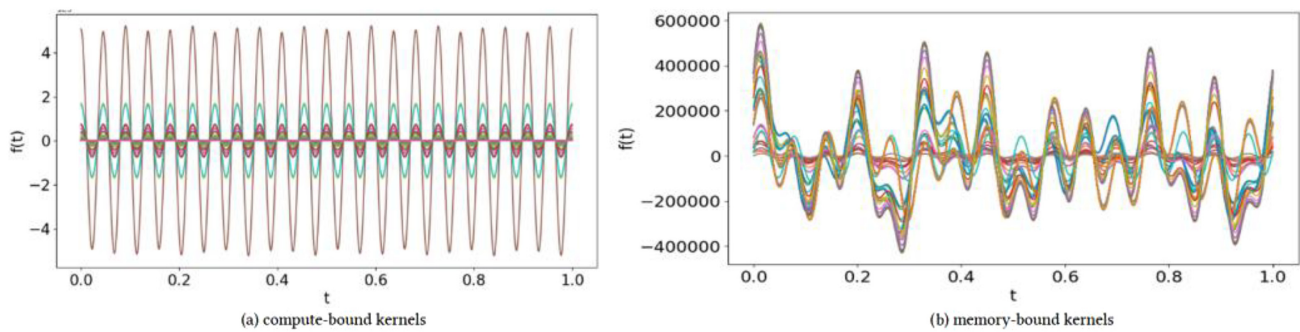

Fig. 14. The Andrews plot for two categories, compute-bound and memory-bound kernels: (a) computebound kernel statistics show a monochrome frequency while (b) memory-bound kernel statistics show existence of different frequency shape and range.

improvement as expected and only $1 \%$ is achieved. However, the performance slowdown is also negligible since the program execution time for CBS is $3 \%$ worse than the serial execution.

One can interpret different achieved speedups because of different combinations of kernel types. As an example, vectorAdd ( $v d)$ is known to be a drastic memory-bound kernel. When this kernel is concurrently run with a compute-bound kernel such as $m m$ or $h s$, there is the possibility of gaining high performance improvement.

For the set [vd,mm,hs,htg], 40\% performance improvement is due to having two memory-bound kernels $v d$ and $h t g$ besides to two compute-bound kernels $m m$ and $h s$. For the set [mm,tr,hs,htg], there is no such balance between compute-bound and memory-bound kernels. Kernel matrixTranspose (tr) is also known to be on the boundary of compute-bound and memory-bound classes. This is also visible in the last experiment including all test kernels. The possibility of having more concurrency is now increased and the performance improvement is more than the previously mentioned experiment.

Comparing the results of CBS with optimal scheduling, CBS acts nearly to the optimal case. Even in some cases such as [mm,vd,tr,htg], the results are the same. This indicates that the proposed metric selection has enough efficiency to provide the most discriminative metrics for classifying kernels into compute-bound or memory-bound classes.

On average, using geometric mean, while $10 \%$ speedup for kernel time is achieved from random scheduling of concurrent kernels, the average speedup for CBS is $18 \%$. The average speedup for the whole program is $9 \%$ for CBS. This presents the importance of taking kernel behavior into account when running multiple kernels concurrently.

\subsection{Discussion}

In order to visualize the efficiency of the selected metrics, Andrews plots [45] are employed. An Andrews plot is a graphical analysis tool to visualize possible structures in high-dimensional multivariate data, such as the mentioned data matrix. The Andrews plot defines a finite Fourier series over the data, i.e., the selected metrics are the coefficients of the Fourier series over different values for $t$. Figure 14 shows the Andrews plot for the two categories of compute-bound and memorybound kernels.

As seen in Figure 14(a), compute-bound class data represents a monochrome frequency shape with a well-ordered simple sine shape. On the other hand, in Figure 14(b), for memory-bound kernels, there exist different types of frequencies for variable frequency ranges. Totally, the discrimination between different types of kernels is clearly shown using this plot.

After applying the proposed feature selection method, the Andrews plots are utilized for the projection of the input data onto two of the results in Table 7 including (a) $\left\{22_{-} l 1 \_r e a d\right.$ throughput, shared_store_throughput, and dram_utilization\} and (b) \{shared_load_throughput, 


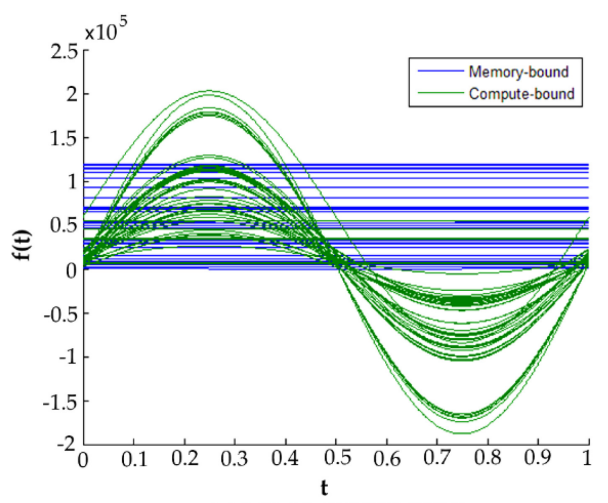

(a) $\{$ L2L1T, SHRST, DRU $\}$

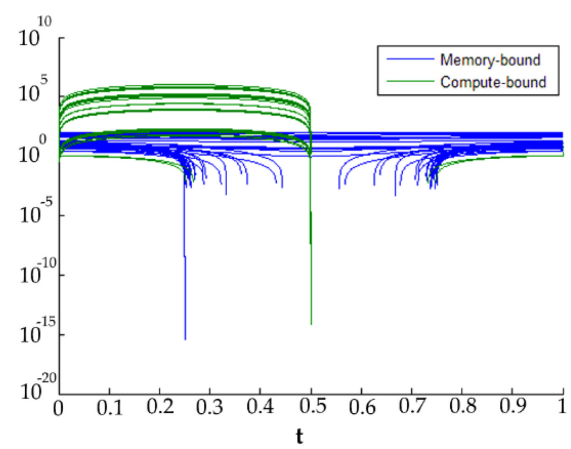

(b) $\{$ SHRLT, SYSMWT, DRU\}

Fig. 15. The results of the classification drawn on an Andrew plot using (a) L2L1T, SHRST, and DRU metrics and (b) SHRLT, SYSMWT, and DRU metrics shows clear discrimination between different type observations. The vertical axis of (b) is drawn in log scale since the difference between values of different classes is extremely high.

sysmem_write_throughput, and dram_utilization\}, as shown in Figure 15. Therefore, the discrimination between the two classes is clearly visible using this visualization method. The selected metrics are considered as the coefficients of the Fourier transformation per kernel class. If the results are well discriminated, the metric subset is consequently selected well.

In Figure 15, for each metric subset, the two classes are drawn in two different colors showing that both subsets are able to provide enough classification accuracy, although there are some small interferences at some points. In this figure, the Fourier transform of the results of classification using subset $\left\{l 2 \_l 1 \_r e a d \_t h r o u g h p u t\right.$, shared_store_throughput, and dram_utilization $\}$ demonstrates high discrimination between members of the two classes. This is similarly true for subset \{shared_load_throughput, sysmem_write_throughput, and dram_utilization\} presented in Figure 15(b).

5.7.1 Classification Granularity. Since there are several different resources available on a GPU, such as registers, different levels of caches, and shared memory, one may think of classifying a kernel into finer classes than the two coarse classes of "memory-bound" and "compute-bound." An example is the convolution kernel, which is a compute-bound kernel. It stresses the GPU by strictly utilizing shared memory. Therefore, it may also belong to a class called "shared-memorybound."

Although the proposed method is defined and described for classifying samples into two classes, it is possible to provide more classes. An extension to the proposed method is a hierarchical classification method. To this end, the proposed method is first applied to select a subset of metrics for classification into two coarse classes, "compute-bound" and "memory-bound." It is then applied to select another subset of metrics to re-classify a classified kernel into a finer class, e.g., "shared-memory-bound" or "register-bound."

Using this approach, the scheduler has all the required information, including the overall and detailed behavior of a given kernel. In the case that the overall runtime behavior of a kernel is sufficient for the scheduler decision, there is no need to continue classification to further levels. If the scheduler needs more detailed information, it can use multiple levels of classification by employing different subsets of metrics.

In addition, since the proposed classifier reads the selected metric values at runtime, it can be designed such that it redoes the classification in a time-slot manner. This means that at the start 
of each time slot, the classifier reads the selected metrics and determines to which of those two classes a kernel belongs. This is also true for other changes in the hardware configuration such as voltage or frequency scaling. To support voltage or frequency scaling, the classifier can be called to refine the classification results.

\section{CONCLUSION AND FUTURE WORK}

The current article presents a method of feature selection to choose the most important metrics for the problem of concurrency-aware kernel classification. New GPU architectures allow the concurrent execution of multiple kernels under the stream capability. However, due to contention on shared resources among different kernels, it is essential to know the runtime behavior of kernels via runtime metrics.

The present study provides a method for selecting the most important metrics for this purpose. This method is a wrapper-based feature selection equipped with Fisher scoring function. Experiments on a Kepler-class architecture show promising results for classification accuracy while the overhead at runtime is kept low. In addition, since any online scheduler may need to read metrics during runtime, it should read as few metrics as possible in order to make a scheduling decision with only a short delay and less overhead.

As an advantage, the proposed method of metric selection is required to be run only once when a new platform is utilized. Hence, the method does not change from application to application on the same platform. This extends the usage of the proposed method in terms of supporting for new application without any prior knowledge. The proposed approach has been evaluated on two GPUs including a NVIDIA GTX760 with a Kepler architecture and a NVIDIA TITAN XP with a Pascal architecture.

The results of scheduling different kernels by utilizing the proposed method of kernel classification confirm the need to do such a classification instead of unaware scheduling. A future work here is to enable the scheduler to divide kernels into a smaller number of thread blocks to run a greater number of smaller concurrent kernels than the original large ones. This will help to have different resources keep busy during runtime.

Although implemented and tested on NVIDIA GPUs, the proposed methodology of metric selection is not limited to specific GPU architectures and products and it can be easily ported to other GPUs. For GPUs from other vendors, if their corresponding runtime systems provide runtime performance counters similar to CUPTI metrics, that can be considered as inputs to the proposed classifier.

Furthermore, any classifier constructed from the results of this method does not need to include all the metrics for classification. There are possible extensions to the current work since newer architectures may provide several new metrics and also because some metrics may become obsolete. It has been shown with evaluating on a modern GPU, TITAN XP. Furthermore, it may be beneficial to look at runtime events instead of metrics. The main purpose of the proposed method is to design an online classifier using CUPTI metrics.

Finally, the results show that to achieve a high accuracy classifier, there is no need to read all metrics and only three and five specific metrics are enough for GTX760 and TITAN XP, respectively.

\section{REFERENCES}

[1] W. Hwu. 2014. What is ahead for parallel computing. f. Parallel Distrib. Comput. 74, 7 (2014), 2574-2581.

[2] D. B. Kirk and W. W. Hwu. 2012. Programming Massively Parallel Processors. Morgan Kaufmann.

[3] T. Li, V. K. Narayana, and T. El-Ghazawi. 2014. Symbiotic scheduling of concurrent GPU kernels for performance and energy optimizations. In Proceedings of the 11th ACM Conference on Computing Frontiers. 36:1-36:10.

[4] NVIDIA. 2018. Streams. In CUDA C Programming Guide PG-02829-001_v9.2. 
[5] F. Zhang, J. Zhai, B. He, S. Zhang, and W. Chen. 2016. Understanding co-running behaviors on integrated CPU/GPU architectures. IEEE Trans. Parallel Distrib. Syst. PP, 99 (2016), 1.

[6] NVIDIA. 2017. CUDA Runtime API v9.2.148.

[7] NVIDIA. 2017. CUPTI User's Guide DA-05679-001_v6.0.

[8] Z. Qi, J. Yao, C. Zhang, M. Yu, Z. Yang, and H. Guan. 2014. VGRIS: Virtualized GPU resource isolation and scheduling in cloud gaming. ACM Trans. Arch. Code Optim. 11, 2 (2014), 17:1-17:25.

[9] Y. Zhang, Z. Xing, C. Liu, C. Tang, and Q. Wang. 2018. Locality based warp scheduling in GPGPUs. Futur. Gener. Comput. Syst. 82 (May 2018), 520-527.

[10] Q.Xu, H. Jeon, K. Kim, W. W. Ro, and M. Annavaram. 2016. Warped-slicer: Efficient intra-SM slicing through dynamic resource partitioning for GPU multiprogramming. In Proceedings of the 2016 ACM/IEEE 43rd Annual International Symposium on Computer Architecture (ISCA'16). 230-242.

[11] Y. Suzuki, Y. Fujii, T. Azumi, N. Nishio, and S. Kato. 2017. Real-time GPU resource management with loadable kernel modules. IEEE Trans. Parallel Distrib. Syst. 28, 6 (2017), 1715-1727.

[12] A. Bakhoda, G. L. Yuan, W. W. L. Fung, H. Wong, and T. M. Aamodt. 2009. Analyzing CUDA workloads using a detailed GPU simulator. In Proceedings of the 2009 IEEE International Symposium on Performance Analysis of Systems and Software. 163-174.

[13] J. Zhong and B. He. 2014. Kernelet: High-throughput GPU kernel executions with dynamic slicing and scheduling. IEEE Trans. Parallel Distrib. Syst. 25, 6 (2014), 1522-1532.

[14] R. Mohammadi, S.-K. Shekofteh, M. Naghibzadeh, and H. Noori. 2016. A dynamic special-purpose scheduler for concurrent kernels on GPU. In Proceedings of the 2016 6th International Conference on Computer and Knowledge Engineering (ICCKE'16). 218-222.

[15] M. Lee, S. Song, J. Moon, J. Kim, W. Seo, Y. Cho, and S. Ryu. 2014. Improving GPGPU resource utilization through alternative thread block scheduling. In Proceedings of the 2014 IEEE 20th International Symposium on High Performance Computer Architecture (HPCA'14). 260-271.

[16] M. E. Belviranli, L. N. Bhuyan, and R. Gupta. 2013. A dynamic self-scheduling scheme for heterogeneous multiprocessor architectures. ACM Trans. Arch. Code Optim. 9, 4 (2013), 57:1-57:20.

[17] S.-K. Shekofteh, H. Noori, M. Naghibzadeh, H. S. Yazdi, and H. Froning. 2017. Enhancing performance of graphics processing units through better scheduling of concurrent kernels. In Proceedings of the 32nd ISC (the International Supercomputing Conference) on High Performance.

[18] X. Mei and X. Chu. 2017. Dissecting GPU memory hierarchy through microbenchmarking. IEEE Trans. Parallel Distrib. Syst. 28, 1 (2017), 72-86.

[19] A. Jog, O. Kayiran, T. Kesten, A. Pattnaik, E. Bolotin, N. Chatterjee, S. W. Keckler, M. T. Kandemir, and C. R. Das. 2015. Anatomy of GPU memory system for multi-application execution. In Proceedings of the 2015 International Symposium on Memory Systems. 223-234.

[20] W. Jia, K. A. Shaw, and M. Martonosi. 2012. Stargazer: Automated regression-based GPU design space exploration. In Proceedings of the 2012 IEEE International Symposium on Performance Analysis of Systems Software. 2-13.

[21] A. Kerr, G. Diamos, and S. Yalamanchili. 2009. A characterization and analysis of PTX kernels. In Proceedings of the 2009 IEEE International Symposium on Workload Characterization (IISWC'09). 3-12.

[22] A. Munshi. 2014. The OpenCL Specification (22nd ed.). Khronos OpenCL Working Group.

[23] K. Asanovic, R. Bodik, B. C. Catanzaro, J. J. Gebis, P. Husbands, K. Keutzer, D. A. Patterson, W. L. Plishker, J. Shalf, S. W. Williams, and K. A. Yelick. 2006. The Landscape of Parallel Computing Research: A View from Berkeley. Technical Report UCB/EECS-2006-183.

[24] E. Konstantinidis and Y. Cotronis. 2015. A practical performance model for compute and memory bound GPU kernels. In Proceedings of the 2015 23rd Euromicro International Conference on Parallel, Distributed and Network-Based Processing (PDP'15). 651-658.

[25] E. Konstantinidis and Y. Cotronis. 2017. A quantitative roofline model for GPU kernel performance estimation using micro-benchmarks and hardware metric profiling. F. Parallel Distrib. Comput. 107 (2017), 37-56.

[26] S. Williams, A. Waterman, and D. Patterson. 2009. Roofline: An insightful visual performance model for multicore architectures. Commun. ACM 52, 4 (2009), 65-76.

[27] S. Mittal and J. S. Vetter. 2014. A survey of methods for analyzing and improving GPU energy efficiency. ACM Comput. Surv. 47, 2 (2014), 19:1-19:23.

[28] B. Wu, G. Chen, D. Li, X. Shen, and J. Vetter. 2015. Enabling and exploiting flexible task assignment on GPU through SM-Centric program transformations. In Proceedings of the 29th ACM on International Conference on Supercomputing. $119-130$.

[29] K. Fukunaga. 1990. Parametric classifiers. In Introduction to Statistical Pattern Recognition (2nd ed). Academic Press, 124-180.

[30] NVIDIA. 2018. CUDA Samples SDK Reference Manual v 9.0.106. 
[31] S. Che, M. Boyer, J. Meng, D. Tarjan, J. W. Sheaffer, S.-H. Lee, and K. Skadron. 2009. Rodinia: A benchmark suite for heterogeneous computing. In Proceedings of the 2009 IEEE International Symposium on Workload Characterization (IISWC'09). 44-54.

[32] W. W. Daniel and others. 1978. Applied Nonparametric Statistics. Houghton Mifflin.

[33] A. Karami, F. Khunjush, and S. A. Mirsoleimani. 2015. A statistical performance analyzer framework for OpenCL kernels on NVIDIA GPUs. f. Supercomput. 71, 8 (2015), 2900-2921.

[34] A. Pattnaik, X. Tang, A. Jog, O. Kayiran, A. K. Mishra, M. T. Kandemir, O. Mutlu, and C. R. Das. 2016. Scheduling techniques for GPU architectures with processing-in-memory capabilities. In Proceedings of the 2016 International Conference on Parallel Architectures and Compilation. 31-44.

[35] Q. Jiao, M. Lu, H. P. Huynh, and T. Mitra. 2015. Improving GPGPU energy-efficiency through concurrent kernel execution and DVFS. In Proceedings of the 13th Annual IEEE/ACM International Symposium on Code Generation and Optimization. 1-11.

[36] G. Wu, J. L. Greathouse, A. Lyashevsky, N. Jayasena, and D. Chiou. 2015. GPGPU performance and power estimation using machine learning. In Proceedings of the 2015 IEEE 21st International Symposium on High Performance Computer Architecture (HPCA'15). 564-576.

[37] S. Muralidharan. 2016. Abstraction and Strategies for Adaptive Programming. Ph.D. dissertation, The University of Utah.

[38] E. Ipek, B. R. de Supinski, M. Schulz, and S. A. McKee. 2005. An approach to performance prediction for parallel applications. In Proceedings of the 11th International Euro-Par Conference on Parallel Processing (Euro-Par'05), J. C. Cunha and P. D. Medeiros (Eds.). Springer, Berlin, 196-205.

[39] B. C. Lee, D. M. Brooks, B. R. de Supinski, M. Schulz, K. Singh, and S. A. McKee. 2007. Methods of inference and learning for performance modeling of parallel applications. In Proceedings of the 12th ACM SIGPLAN Symposium on Principles and Practice of Parallel Programming. 249-258.

[40] A. Majumdar, G. Wu, K. Dev, J. L. Greathouse, I. Paul, W. Huang, A. K. Venugopal, L. Piga, C. Freitag, and S. Puthoor. 2015. A taxonomy of GPGPU performance scaling. In Proceedings of the 2015 IEEE International Symposium on Workload Characterization (IISWC'15).

[41] R. Sheikhpour, M. A. Sarram, S. Gharaghani, and M. A. Z. Chahooki. 2017. A survey on semi-supervised feature selection methods. Pattern Recogn. 64, C (2017), 141-158.

[42] J. Miao and L. Niu. 2016. A survey on feature selection. Procedia Comput. Sci. 91, Supplement C (2016), 919-926.

[43] A. Jain and D. Zongker. 1997. Feature selection: Evaluation, application, and small sample performance. IEEE Trans. Pattern Anal. Mach. Intell. 19, 2 (1997), 153-158.

[44] R. A. Horn and C. R. Johnson. 1985. Variational characterization of eigenvalues of Hermitian matrices. Matrix Analysis 67, 3 (1985), 212.

[45] D. F. Andrews. 1972. Plots of high-dimensional data. Biometrics 28, 1 (1972), 125-136.

Received May 2018; revised November 2018; accepted November 2018 